\title{
The cis and trans effects of the risk variants of coronary artery disease in the Chr9p21 region
}

\author{
Wei Zhao ${ }^{1 *}$, Jennifer A Smith ${ }^{1}$, Guangmei Mao ${ }^{1}$, Myriam Fornage ${ }^{2}$, Patricia A Peyser ${ }^{1}$, Yan V Sun ${ }^{3}$, \\ Stephen T Turner ${ }^{4}$ and Sharon LR Kardia ${ }^{1}$
}

\begin{abstract}
Background: Recent genome-wide association studies (GWAS) have shown that single nucleotide polymorphisms (SNPs) in the Chr9p21 region are associated with coronary artery disease (CAD). Most of the SNPs identified in this region are non-coding SNPs, suggesting that they may influence gene expression by cis or trans mechanisms to affect disease susceptibility. Since all cells from an individual have the same DNA sequence variations, levels of gene expression in immortalized cell lines can reflect the functional effects of DNA sequence variations that influence or regulate gene expression. The objective of this study is to evaluate the functional consequences of the risk variants in the Chr9p21 region on gene expression.
\end{abstract}

Methods: We examined the association between the variants in the Chr9p21 region and the transcript-level mRNA expression of the adjacent genes (cis) as well as all other genes across the whole genome (trans) from transformed beta-lymphocytes in 801 non-Hispanic white participants from The Genetic Epidemiology Network of Arteriopathy (GENOA) study.

Results: We found that the CAD risk variants in the Chr9p21 region were significantly associated with the mRNA expression of the ANRIL transcript ENST00000428597 ( $p=8.58 \mathrm{e}-06)$. Importantly, a few distant transcripts were also found to be associated with the variants in this region, including the well-known CAD risk gene ABCA1 ( $p=1.01 \mathrm{e}-05)$. Gene enrichment testing suggests that retinol metabolism, N-Glycan biosynthesis, and TGF signaling pathways may be involved.

Conclusion: These results suggest that the effect of risk variants in the Chr9p21 region on susceptibility to CAD is likely to be mediated through both cis and trans mechanisms.

Keywords: GENOA, Gene expression, SNP, CAD, Chr9p21

\section{Background}

Atherosclerosis is a condition in which plaque builds up within the walls of the coronary arteries that can lead to a decreased supply of oxygen-rich blood to the heart muscle. Over time, the accumulation of plaques within the coronary arteries can weaken the heart muscle and lead to heart failure and arrhythmias. The end result is coronary artery disease (CAD), which is the leading cause of death in the United States and many western countries [1-3]. Genetic epidemiological studies of families and twins have suggested that genetic mechanisms contribute to CAD susceptibility after adjusting for other major non-genetic risk

\footnotetext{
* Correspondence: zhaowei@umich.edu

'Department of Epidemiology, University of Michigan, Ann Arbor, MI, USA Full list of author information is available at the end of the article
}

factors [4]. In particular, genome-wide association studies (GWAS) of common SNPs have found that single nucleotide polymorphisms (SNPs) in the Chr9p21 region are associated with CAD in populations with European ancestry [5-10]. This association was later replicated in many other ethnicities, including Asians and Hispanics [11-13]. In addition to CAD, this locus has also been found to be associated with many other cardiovascular traits, including stroke [14], aneurysm [15,16] and peripheral artery disease [17]. This suggests the Chr9p21 region has broad effects on the cardiovascular system.

The Chr9p21 haplotype block is a 113.5-kb region from base pair 22,012,420 to 22,125,915 on chromosome 9 that contains multiple SNPs that are in high linkage disequilibrium [18]. Even though the association between CAD and 
the variants in the Chr9p21 region is consistent across many different studies and different ethnicities [5-13], the fact that the SNP associated with CAD in GWAS does not fall within gene boundaries makes the interpretation of the association difficult. Moreover, the association between the Chr9p21 region and CAD is independent of any traditional risk factors, such as hypertension or lipid profile [5-7]. All of this evidence suggests that the Chr9p21 region affects atherosclerosis through a novel mechanism that is yet to be discovered.

Genetic variants can affect RNA expression through either cis or trans mechanisms or both. In this paper, the cis effect refers to the effects of the genetic variants of the Chr9p21 region on the genes within the same region. The effects of the variants on all other genes are referred to as a trans effect. The cis effect of the Chr9p21 region has been investigated by several studies. There are four genes that are adjacent to the region of chromosome 9p21, which are CDKN2A, CDKN2B, ANRIL and C9orf53. $C D K N 2 A$ and $C D K N 2 B$ are cyclin-dependent kinase inhibitors and are known tumor suppressors [19]. ANRIL, also known as $C K D N 2 B-A S$, is a non-coding RNA [20] and $C 9$ orf53 is an open reading frame. The functions of ANRIL and C9orf53 are not clear. Several studies have examined the genetic effect of the identified risk variants in this region on the abundance of mRNA level of these genes in a variety of cells and tissues [21-27]. The majority of these studies found robust associations between ANRIL expression and the Chr9p21 genotypes. All of them suggest an important role for ANRIL in the susceptibility of CAD. However, all of these studies have examined only a small number of CAD risk SNPs. There is no evidence so far that the identified risk SNPs are the true functional SNPs. Thus, a larger range of survey, including all of the common and rare variants in the region, is needed. Moreover, alternative splicing plays an important role in the gene expression. Investigating transcript level mRNA abundance, as opposed to the whole gene level, would lend insight into understanding the function. Lastly, despite the importance of the Chr9p21 region, there is no study that particularly investigated the trans effects of the region, which may play a more proximal role on atherosclerosis than the cis effects $[28,29]$.

The Genetic Epidemiology Network of Arteriopathy Study (GENOA), a network in the Family Blood Pressure Program [30], is a community-based study that recruited sibships with at least two adults with clinically diagnosed essential hypertension before age 60. GENOA has genome wide measurement of exon-level gene expression profiles in immortalized beta-lymphocyte cell lines and genomewide genotypes on about 800 individuals, which provides a unique epidemiological opportunity to evaluate both the cis and the trans effect of the variation in the Chr9p21 region on gene expression. Immortalized beta-lymphocyte cell lines are not the most proximal tissue for examining the atherosclerotic process, however, they provide an efficient and robust way to examine the effects of DNA sequence variation on variation in gene expression since the environmental conditions of the cells are the same across individuals (i.e. environmental variation is minimized). We also used a SNP-set based association method [31] to examine the effects of all variants in the $9 \mathrm{p} 21$ region at once for transcriptome wide gene expression analysis.

\section{Methods \\ Sample}

The GENOA study is a community-based study of sibships that aims to identify genes influencing blood pressure $[30,32]$. Sibships with at least two adults with clinically diagnosed essential hypertension before age 60 were recruited. All other members of the sibship were invited to participate regardless of their hypertension status. All subjects came from the Rochester, MN field center and were non-Hispanic whites of European descent. Among the participants, 801 individuals had both genome-wide SNP data and gene expression data. The GENOA study was approved by the University of Michigan Health Sciences and Behavioral Sciences Institutional Review Board and the Mayo Clinic Institutional Review Board. Each participant gave written informed consent.

\section{Genotyping and quality control of GWAS SNPs}

GENOA participants were first genotyped using the Affymetrix Genome-wide Human SNP Array 6.0 platform. The samples whose genotyping was not successful on the Affymetrix 6.0 platform were re-genotyped using the Illumina Human1M-DUO BeadChip (Illumina, 2010). For each platform, participants were excluded if they had an overall SNP call rate $<95 \%$ or sex mismatch between genotypic and phenotypic measurement. SNPs were excluded if they had an unknown chromosomal location or a call rate less than $95 \%$. These quality control filters resulted in 668,293 SNPs in 1,386 subjects available from the Affymetrix platform and 893,545 SNPs in 123 subjects from the Illumina platform for analysis.

Principal component analysis was conducted as an additional quality control step to identify and remove samples with outlying genotype profiles. After forty-five outliers were identified and excluded, a total of 1,464 individuals had genotype data available for analysis.

For this analysis, imputation was done using the European reference panel (87 CEU, 98 TSI, 89 GBR, 93 FIN, 66 MXL, 55 PUR) from the 1000 Genome Project. Prior to imputation, SNPs were additionally filtered for minor allele frequency (MAF) $\geq 0.01$ and Hardy-Weinberg P-value $\geq$ 0.001. MACH (v1.0.16) and Minimac (v 4.4.3) were used to impute genotypes of unmeasured markers, up to total of 
21,880,364 SNPs. $\mathrm{MaCH}$ was used in the pre-phasing stage, and Minimac was used in the imputation stage.

After the above steps, 180 SNPs within the region of Chr9p21 (chr9: 22,012,420-22,125,915 bp) that had imputation quality score $\mathrm{R}^{2}$ greater than 0.9 and MAF greater than 0.0025 were selected for analysis.

\section{Gene expression profiling and quality control}

RNA samples from cell lines established from peripheral blood samples by Epstein-Barr virus transformation were extracted using standard protocols. RNA quality was assessed using the Agilent 2100 Bioanalyzer (Agilent Technologies Inc., Foster City, CA) and quantified by spectrophotometry using the Nanodrop ND-1000 (Nanodrop Inc., Wilmington, DE). All RNA samples used in the present study yielded both anA260/A280 absorbance ratio greater than 2.0 and a RNA Integrity Number (RIN) $\geq 8$.

One $\mu \mathrm{g}$ of RNA was labeled using the WT Expression labeling assay (Applied Biosystems/ Ambion, Foster City, $\mathrm{CA}$ ) including the labeling controls from the GeneChip Eukaryotic Poly-A RNA Control Kit (Affymetrix, Santa Clara, CA). Each step of the labeling protocol was monitored using the Agilent 2100 Bioanalyzer or the Nanodrop spectrophotometer, as recommended by the manufacturer. Hybridization buffer, Eukaryotic Hybridization Controls, and OligoB2 controls were added to the cDNA fragments just prior to hybridization to the Affymetrix Human Exon 1.0 ST Array. Hybridization was performed at $45^{\circ} \mathrm{C}$ for 17 hours. Following hybridization, the chips were washed and stained with a phycoerythrin-strepavidin conjugate and were scanned at the excitation wavelength of $488 \mathrm{~nm}$.

Array quality control was performed using Affymetrix Expression Console $^{\mathrm{m}}$ ( $\mathrm{v}$ 1.1) at the transcript level using core-level probe sets. All array images passed visual inspection. Hybridization controls were all present with signal increases following concentration. Labeling control signal strengths followed the order Lys $<$ Phe $<$ Thr $<$ Dap. Signal intensity plots were examined for raw and processed data to identify outliers. Summarization QC metrics, including relative log expression (RLE), positive vs. negative ROC AUC and MAD-residual mean, were all within the parameters suggested by Affymetrix.

Raw intensity data were processed using Multiple Exon Array Preprocessing (MEAP) software. The PMBayesBG method was used for background correction. This was followed by quantile normalization, expression summarization and $\log _{2}$ transformation [33]. The expression summarization is multi-dimensional depending on the user defined data type, which includes probeset, exon, spliced variant or gene. The transcript (spliced variant) level summary was used in this study. Annotation version 64 was used for our expression summarization. Exon expression intensities were summarized directly from probe- level expression values and MEAP annotation. Expression summarizations for transcripts were calculated from exon expression by linear algebra based on transcript records in external databases. If the probes targeting an exon did not have strong signals and thus caused low or no expression of the exon, it may have resulted in a negative algebraic solution. The transcript's expression values were denoted as missing in that case. If a transcript had a missing rate greater than $10 \%$, it was excluded from the analysis.

Principal component analysis on the exon level expression data was used to identify possible outliers, and none were identified. We also verified sex assignment of the samples by examining expression levels of genes on the $\mathrm{Y}$ chromosome. Two individuals were excluded due to sex mismatch. There were a total of 875 individuals who had gene expression data. Among them, 801 individuals had both gene expression and SNP data.

\section{Statistical methods}

To evaluate the cis effect, the association between each SNP in the Chr9p21 region and the candidate transcripts from the four adjacent genes were tested using linear mixed models with each transcript as the outcome, SNP as the predictor, and age, sex and batch as the covariates. Familial correlation was adjusted by the random effect. SNPs were coded additively. Since there were 8 transcripts and 180 SNPs in total, the Bonferroni corrected $\mathrm{p}$ value for each transcript was 3.47e-5. Among those SNPs, we also specifically examined six known CAD risk variants: rs7865618, rs10811650, rs1333040, rs10757274, rs2383206, and rs1333045 [34].

In order to increase our power to detect trans effects, SNP-set (Sequence) Kernel Association Test (SKAT) [31] was used to test the association between gene expression and the SNP-set within the Chr9p21 region. SKAT allows different weights to be assigned to different variants according to a hypothesis or existing knowledge. The default weight follows a Beta $(1,25)$ function, which assumes rare causal variants have larger effects, and thus assigns larger weights to rare variants. Beta(1,1) assumes all of the causal variants have similar effect sizes and assigns equal weights to all the variants. Beta $(0.5,0.5)$ produces the Madsen \& Browning weights that have been a popular choice, which also assigns weight based on the minor allele frequency (MAF) but does not up-weight rare variants as much as the Beta(1,25) function [35]. In order to assess the effect of both rare variants and common variants, we applied all three weighting schemes to the analysis. To adjust for familial correlation, we implemented a two-step approach. First, the transcript level gene expression was adjusted for familial correlation by a linear mixed model and the random residual was saved. Second, SKAT was used to test the association between the residual from the previous step and all the SNPs within the Chr9p21 region, with batch, 
age and sex as the covariates. SNPs were coded additively. A total of 35,477 transcripts across the whole genome were analyzed, false discovery rate (FDR) at $10 \%$ was applied to adjust for multiple testing. To identify trans expression quantitative trait loci (eQTLs) at the SNP level, we followed up each SKAT association that had FDR $\mathrm{q}<0.1$ with single SNP analysis using all of the SNPs in the Chr9p21 region. The statistical model was the same as the model used for the cis effect analysis.

Since the transcripts across the genome are highly correlated, it is often too conservative to simply apply a stringent criterion for association. Thus we also performed gene set enrichment testing for transcripts at least nominally associated with the Chr9p21 region ( $\mathrm{p}$-value $<0.01$ ) in the $\operatorname{Beta}(1,25)$, Beta $(1,1)$ and $\operatorname{Beta}(0.5,0.5)$ SKAT analyses. DAVID [36] was used for gene set enrichment testing, in which the background was set as the total of 35,477 transcripts. The p-value of each gene set was calculated using a modified Fisher exact test.

All statistical analyses were performed with $\mathrm{R}$ statistical environment version 2.9.0 from $\mathrm{R}$ Project (http:// www.r-project.org/). The authors had full access to the data and take responsibility for its integrity. All authors have read and agree to the manuscript as written.

\section{Results}

There were 801 individuals who had both expression profiles and SNP data. There were 337 men and 464 women, and the mean age was 59.2 years. The majority of the participants $(75.9 \%)$, by study design, had hypertension. There were 82 subjects with a history of coronary artery disease.

The Chr9p21 haplotype block is a 113.5-kb region from base pair 22,012,420 to 22,125,915 on chromosome 9 [18]. The adjacent four genes of this region are ANRIL, $C D K N 2 A, C D K N 2 B$ and C9orf53 and the available transcripts are presented in the first column in Table 1 . In our data, ANRIL has 2 transcripts, $C D K N 2 A$ has 3 transcripts, $C D K N 2 B$ has 2 transcripts and C9orf53 has 1 transcript. The effect of each of the $180 \mathrm{Chr} 9 \mathrm{p} 21$ SNPs on each transcript was evaluated. The SNP with the strongest association with each transcript is shown in Table 1 . The Bonferroni corrected alpha level is 3.47e-5. Among the 8 SNPs shown in Table 1, only the top SNP for the ANRIL transcript ENST00000428597 was significantly associated after Bonferroni correction. No other transcripts were significantly associated with any SNPs in the Chr9p21 region.

Next, we plotted the p value of the association between the ANRIL transcript and each SNP against the chromosome position in Figure 1A. There were a total of 18 SNPs that were significantly associated with the mRNA expression of the ANRIL transcript ENST00000428597 at the Bonferroni corrected $\mathrm{p}$ value. As shown in Figure 1A, almost all the SNPs that were associated with the ANRIL transcript are in linkage disequilibrium with the top SNP rs7865618. The mRNA expression level of ANRIL transcript ENST00000428597 was plotted against SNP rs7865618 as shown in Figure 1B. One increased copy of $\mathrm{A}$ in rs7865618 is associated with a $7 \%$ increase in the ANRIL transcript ENST00000428597 on the $\log _{2}$ scale, which corresponds to a $5 \%$ increase on the original scale.

We also specifically examined the association between known CAD risk SNPs in the Chr9p21 region and the ANRIL transcript ENST00000428597, and the results are summarized in Table 2. Our top SNP, rs7865618, is included in the group of CAD risk SNPs. Moreover, all of the six candidate SNPs showed a trend of association with this transcript, and three were significantly associated at a nominal level $(p=0.05)$. Because we know that there is strong linkage disequilibrium in the Chr9p21 region, we examined the correlation among the six candidate SNPs in our dataset and they were all correlated with each other (absolute $\mathrm{r}$ ranges from 0.538 to 0.976 ). For all of the

Table 1 Top SNPs associated with each transcript

\begin{tabular}{|c|c|c|c|c|c|c|c|}
\hline Transcript ID & Top SNP & Coded allele & Non coded allele & Coded allele frequency & Parameter estimate & SE & P-value \\
\hline \multicolumn{8}{|l|}{ ANRIL } \\
\hline ENST00000428597 & rs7865618 & A & G & 0.569 & 0.07 & 0.016 & $8.58 \mathrm{e}-06$ \\
\hline ENST00000422420 & rs1759417 & C & $\mathrm{T}$ & 0.834 & -0.08 & 0.045 & 0.088 \\
\hline \multicolumn{8}{|l|}{ C9orf53 } \\
\hline ENST00000441769 & rs662463 & G & A & 0.896 & 0.07 & 0.024 & 0.006 \\
\hline \multicolumn{8}{|l|}{ CDKN2A } \\
\hline ENST00000380151 & rs647188 & T & G & 0.891 & -0.07 & 0.055 & 0.199 \\
\hline ENST00000361570 & rs647188 & $\mathrm{T}$ & G & 0.891 & -0.07 & 0.045 & 0.121 \\
\hline ENST00000304494 & rs647188 & $\mathrm{T}$ & G & 0.891 & -0.06 & 0.047 & 0.209 \\
\hline \multicolumn{8}{|l|}{ CDKN2B } \\
\hline ENST00000380142 & rs2095144 & G & $A$ & 0.707 & 0.03 & 0.012 & 0.030 \\
\hline ENST00000276925 & rs1333040 & T & C & 0.605 & 0.02 & 0.011 & 0.070 \\
\hline
\end{tabular}


A

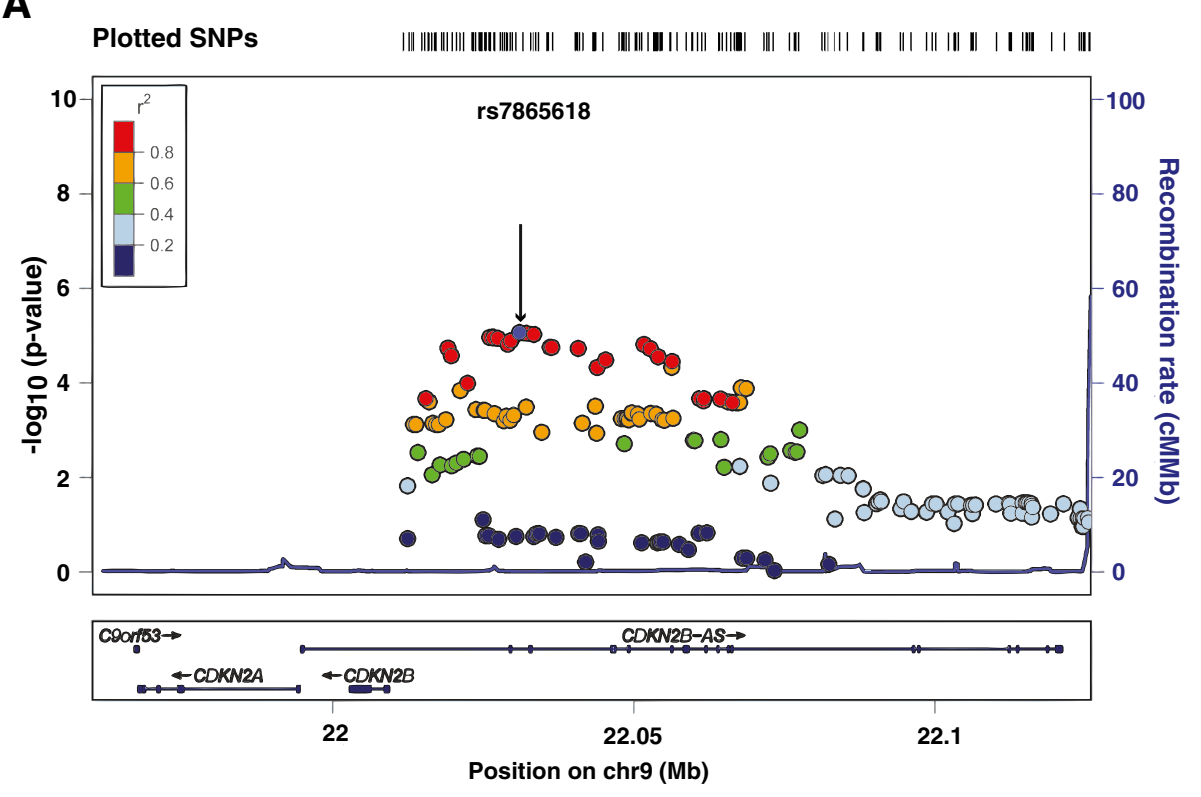

B

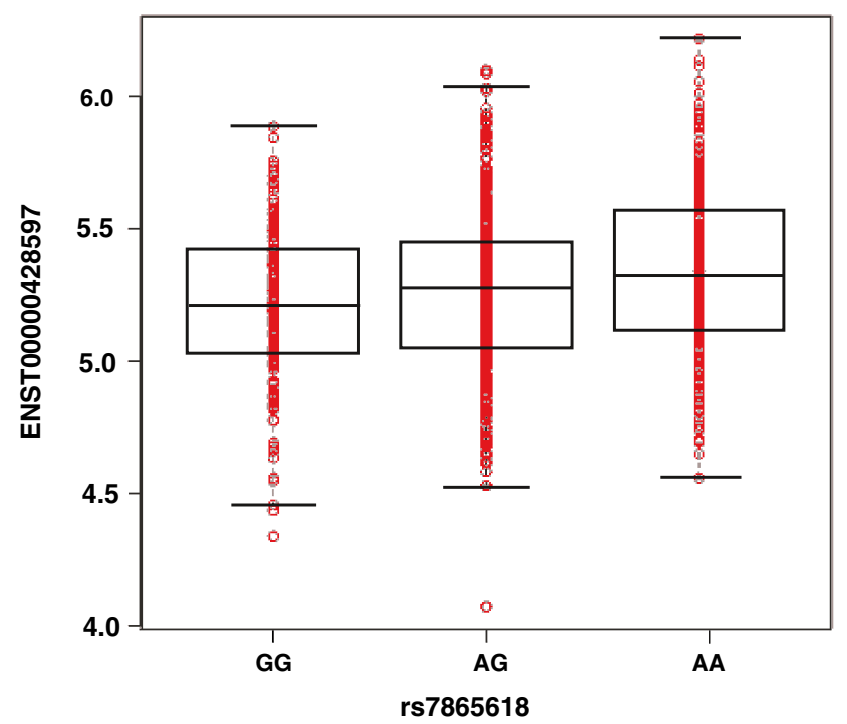

Figure 1 The association between the ANRIL transcript ENST00000428597 and the SNPs in the Chr9p21 region. A. The relationship between the ANRIL transcript ENST00000428597 and the SNPs in the Chr9p21 region. SNPs are plotted by chromosomal position against the -log(p-value) of their association with the ANRIL transcript ENST00000428597. The most significant SNP is shown as a purple circle, and indicated by an arrow. The SNPs surrounding the most significant SNP are color-coded to reflect their linkage disequilibrium with this SNP as shown in the inset (taken from pairwise $r^{2}$ values from the 1000 Genomes Project EUR database). Genes and the position of exons, as well as the direction of transcription, are noted below the plots (data from UCSC Genome Browser). B. Plot of the mRNA expression of ANRIL transcript ENST00000428597 and the top SNP rs7865618. The mRNA expression level ( $\log _{2}$ scale) is plotted against each genotype and is shown in red. The black boxplot is added on top of the scatterplot to show the median and interquartile range of expression level for each genotype.

CAD risk SNPs in the Chr9p21 region, the risk variant always increases the mRNA expression of the transcript.

In order to better understand the potential importance of genetic variation in this chromosomal region on the larger metabolic processes underlying the atherosclerotic processes, we used the SKAT method to perform a transcriptome-wide assessment of the Chr9p21 region's trans effect. The top transcripts that were associated with the Chr9p21 region through trans effects are listed in Table 3. When the $\operatorname{Beta}(1,25)$ weight was used, four transcripts from four genes showed significant association with the Chr9p21 region. They were DUT 
Table 2 The association between ANRIL ENST00000428597 and known CAD risk variants

\begin{tabular}{|c|c|c|c|c|c|c|c|}
\hline CAD risk SNP & Risk allele & Coded allele & Non coded allele & Coded allele frequency & Parameter estimate & SE & P-value \\
\hline rs7865618 & A & A & G & 0.569 & 0.070 & 0.0155 & $8.58 \mathrm{e}-06$ \\
\hline rs10811650 & G & G & A & 0.490 & 0.043 & 0.0154 & 0.0058 \\
\hline rs1333040 & $\mathrm{T}$ & $\mathrm{T}$ & C & 0.605 & 0.028 & 0.0158 & 0.0755 \\
\hline rs10757274 & G & G & A & 0.490 & 0.030 & 0.0152 & 0.0522 \\
\hline rs2383206 & G & G & A & 0.504 & 0.033 & 0.0153 & 0.0345 \\
\hline rs1333045 & C & C & $\mathrm{T}$ & 0.543 & 0.030 & 0.0158 & 0.0588 \\
\hline
\end{tabular}

(ENST00000331200), EIF1AY (ENST000000361365), CASP14 (ENST00000427043), and ABCA1 (ENST000 000374736). When the $\operatorname{Beta}(1,1)$ and $\operatorname{Beta}(0.5,0.5)$ weighting schemes were used, the top transcript from those two tests was the same for the same gene: DHRS9 (ENST00000412271). However, this transcript was not significant after controlling for multiple testing. As a comparison, we also provide the SKAT test results for the ANRIL transcript (ENST00000428597) that has shown significant association with multiple SNPs in the Chr9p21 region. This transcript was significant in the SKAT test when the weights $\operatorname{Beta}(1,1)$ or $\operatorname{Beta}(0.5,0.5)$ were applied, but was not significant when the weight $\operatorname{Beta}(1,25)$ was applied. As shown in Table 3, the transcript DHRS9 (ENST00000412271) was more significant than the ANRIL transcript, which is known to be associated with the Chr9p21 region. Thus, this gene was also included for the following single SNP analysis.

Next, we looked at the associations between transcripts and single SNPs using the identified top trans-effected transcripts and all of the SNPs in the Chr9p21 region. The top SNP for each transcript is listed in Table 4. As shown in Table 4, the top SNPs (rs16923583, rs41386451, rs16905597) for DUT, EIF1AY, CASP14 and ABCA1 were all rare variants (MAF approximately 0.005). Moreover, we found that the top 4 SNPs for all four of these genes were the same rare variants (rs16923583, rs41386451, rs16905597 and rs73650008), although the rank of the associations are different across genes (Additional file 1: Table S1). The top SNP (rs4977756) for the transcript DHRS9 was a common SNP (MAF $=0.44)$, which was also correlated with all of the CAD risk SNPs in the Chr9p21 region (absolute $r$ ranges from 0.58 to 0.83 ). These results are consistent with the underlying assumptions of the three different SAKT weighting schemes that have been used in this study. One common problem for rare variant analysis is that the detected associations are often driven by one or a few samples. That is what we observed in our study as well (Additional file 2: Figure S1). Thus, functional studies are needed to validate our findings.

To further examine the trans effect in multiple tissues and across cohorts, we searched the above identified associations between gene expression levels and Chr9p21 SNPs (SNP rs4977756 with DHRS9 as well as the 4 rare variants with ABCA1, CASP14, DUT, EIF1AY) in two public eQTL databases, GENe Expression VARiation (Genevar) [37] and the Genotype-Tissue Expression project (GTEx) [38]. We preferentially focused on the HapMap3 project in Genevar

Table 3 The top transcripts associated with the Chr9p21 region from the SKAT test

\begin{tabular}{|c|c|c|c|c|c|c|c|}
\hline \multirow[t]{3}{*}{ Transcript } & \multirow[t]{3}{*}{$\mathbf{N}$} & \multirow{2}{*}{$\begin{array}{l}\text { SKAT } \\
\text { Beta(1,25) }\end{array}$} & \multirow{2}{*}{$\begin{array}{l}\text { SKAT } \\
\operatorname{Beta}(1,1)\end{array}$} & \multirow{2}{*}{$\begin{array}{l}\text { SKAT } \\
\text { Beta }(0.5,0.5)\end{array}$} & \multirow{3}{*}{$\begin{array}{l}\text { FDR } \\
\text { q value }\end{array}$} & \multirow[t]{3}{*}{ Gene } & \multirow[t]{3}{*}{ Location } \\
\hline & & & & & & & \\
\hline & & $P$ value & $P$ value & $P$ value & & & \\
\hline \multicolumn{8}{|c|}{ Top transcripts when SKAT_Beta $(1,25)$ was used } \\
\hline ENST00000331200 & 800 & $8.19 \mathrm{e}-07$ & 0.905 & 0.416 & 0.0276 & DUT & $15 q 21$ \\
\hline ENST00000361365 & 781 & $1.55 \mathrm{e}-06$ & 0.762 & 0.360 & 0.0276 & EIFTAY & Yq11 \\
\hline ENST00000427043 & 796 & $5.79 e-06$ & 0.909 & 0.490 & 0.0685 & CASP14 & $19 \mathrm{p} 13$ \\
\hline ENST00000374736 & 801 & $1.01 \mathrm{e}-05$ & 0.719 & 0.387 & 0.0893 & $A B C A 1$ & $9 q 31$ \\
\hline \multicolumn{8}{|c|}{ Top transcript when SKAT_Beta $(1,1)$ was used } \\
\hline ENST00000412271 & 749 & 0.608659 & $3.05 e-05$ & $3.38 \mathrm{e}-05$ & 0.9998 & DHRS9 & $2 q 31$ \\
\hline \multicolumn{8}{|c|}{ Top transcript when SKAT_Beta $(0.5,0.5)$ was used } \\
\hline ENST00000412271 & 749 & 0.608659 & $3.05 e-05$ & $3.38 \mathrm{e}-05$ & 0.9998 & DHRS9 & $2 q 31$ \\
\hline \multicolumn{8}{|l|}{ cis transcript } \\
\hline ENST00000428597 & 801 & 0.47 & 0.002013 & 0.001907 & 1 & ANRIL & $9 p 21$ \\
\hline
\end{tabular}


Table 4 The top SNPs associated with the trans-effected transcripts identified by SKAT

\begin{tabular}{lllllllll}
\hline Gene & Transcript & Top SNP & Coded allele & Non coded allele & MAF & Beta & SE & P value \\
\hline DUT & ENST00000331200 & rs16923583 & T & A & 0.9944 & 1.07 & 0.187 & $2.14 \mathrm{e}-08$ \\
EIF1AY & ENST00000361365 & rs41386451 & A & C & 0.9952 & 1.77 & 0.377 & $3.66 \mathrm{e}-06$ \\
CASP14 & ENST00000427043 & rs16923583 & T & A & 0.9944 & 1.25 & 0.236 & $1.95 \mathrm{e}-07$ \\
ABCA1 & ENST00000374736 & rs16905597 & G & A & 0.9951 & -0.58 & 0.108 & $1.65 \mathrm{e}-07$ \\
DHRS9 & ENST00000412271 & rs4977756 & A & G & 0.5635 & 0.35 & 0.089 & $9.84 \mathrm{e}-05$ \\
\hline
\end{tabular}

and heart related tissue in GTEx. At alpha level of 0.05, DHRS was found to be associated with rs4977756 in transformed B-lymphoblastoid cells (TBLs) in European Americans (CEU, $\mathrm{p}=0.017$ ) and the direction of effect was consistent with the observed effect direction in GENOA. However, there was not a significant association between rs4977756 and any heart related tissue in GTEx. Only two of the four rare variants, rs16923583 and rs16905597, were available in a small number of non-CEU populations in Genevar, and the associations were not significant. The other two rare variants were not available in either of the databases.

As an alternative, we looked up the association between the six known common Chr9p21 CAD risk SNPs (Table 2) and the genes DUT, EIF1AY, CASP14 and ABCA1. In the Genevar database, we found that rs10811650 was associated with $A B C A 1$ expression $(p=0.015)$ in TBLs in CEU and CASP14 expression $(p=0.0017)$ in an African population (LWK). Also, rs1333040 was associated with DUT expression $\left(p=6.0 \times 10^{-4}\right)$ in a separate African population (YRI). Moreover, in the GTEx database, $A B C A 1$ was associated with four SNPs (rs1333045: $\mathrm{p}=0.009$; rs2383206: $\mathrm{p}=0.002 ; \mathrm{rs} 10757274: \mathrm{p}=0.003 ;$ rs7865618: $\mathrm{p}=0.008)$ in 74 artery aorta samples. EIF1AY was associated with the same set of SNPs in 87 heart left ventricle samples (rs1333045: $\mathrm{p}=0.006 ;$ rs2383206: $\mathrm{p}=0.02$; rs10757274: $\mathrm{p}=0.005$; rs7865618: $\mathrm{p}=0.007$ ). This association between Chr9p21 SNPs and gene expression in left ventricular and aorta tissue provide suggestive evidence that the Chr9p21 region has trans effects in relevant tissues for several of the genes that we have identified.

To discover the potential biological importance of trans-effected genes, we used the KEGG Pathway for gene enrichment analysis. For the top transcripts in $\operatorname{Beta}(1,25)$, there was no significantly enriched gene set with $\mathrm{p}$-value $<0.1$. However, for the top transcripts in $\operatorname{Beta}(1,1)$ and $\operatorname{Beta}(0.5,0.5)$, there were two and three gene sets, respectively, that were enriched at a nominal p-value $<0.1$. As shown in Table 5, the enriched pathways include retinol metabolism and TGF- $\beta$ signaling for $\operatorname{Beta}(1,1)$, and all of the same pathways plus N-Glycan biosynthesis for $\operatorname{Beta}(0.5,0.5)$. The top enriched pathway is retinol metabolism, in which DHRS9 (ENST00000432060, ENST00000412271), ADH1 (ENST00000394887), DHRS4L2
(ENST00000335125), LRAT (ENST00000336356) and CYP26B1 (ENST00000001146) are significantly associated with the Chr9p21 region. One of the two transcripts of DHRS9 (ENST00000412271) is the most significant transeffect gene in $\operatorname{Beta}(1,1)$ and $\operatorname{Beta}(0.5,0.5)$.

\section{Discussion}

Even though the Chromosome 9p21 region has been implicated as a hotspot associated with CAD, it continues to be a challenge to understand the immediate biological and functional effects of variants in that region. Studying the association between genetic variants and gene expression variation is valuable for connecting variation at the DNA sequence level to that at the RNA level. In this study, we used a large family-based cohort where both genotype and gene expression were measured on the same individuals to evaluate both the cis and the trans effects of the genetic variants in the Chr9p21 region on the RNA expression at the transcript level. It was found that most CAD associated SNPs in the Chr9p21 region were highly associated with the expression of the ANRIL transcript ENST00000428597. In addition, we found that the Chr9p21 region was also associated with gene expression of a few distant functional genes and pathways. These results suggest that the genetic effect of risk variants in Chr9p21 region on susceptibility to $\mathrm{CAD}$ is likely to be mediated through both cis and trans effects.

There have been numerous studies looking at the local functional impact of the Chr9p21 variants on gene expression in a variety of tissues and cells [21-27]. All of these studies consistently showed the CAD risk variants in the Chr9p21 region were associated with ANRIL expression and have very little effect on the other local transcripts. For example, earlier studies found that a CAD risk variant was associated with reduced expression levels of ANRIL in purified peripheral blood T-cells, whole blood cells and vascular smooth muscle cells [22,24,27]. However, these studies only examined ANRIL expression at the whole gene level and did not take alternative splicing into account, which could have masked the true effect. Our study examined the mRNA expression at the transcript level and found that SNP rs7865618, which is a CAD risk variant [6,34], was associated with the gene expression of the ANRIL long transcript ENST00000428597. The risk variant 
Table 5 Gene set enrichment testing of KEGG pathway for nominally significant transcripts in $B$ eta(0.5,0.5) and $\operatorname{Beta}(\mathbf{1}, \mathbf{1})(\mathrm{p}$-value $<0.1)$

\begin{tabular}{|c|c|c|c|c|}
\hline & Term & Number of genes & $P$ value & Enriched transcript \\
\hline \multirow[t]{3}{*}{$\operatorname{Beta}(0.5,0.5)$} & hsa00830:Retinol metabolism & 5 & 0.016 & $\begin{array}{l}\text { ENST00000394887, ENST00000001146, ENST00000432060, } \\
\text { ENST00000412271, ENST00000336356 }\end{array}$ \\
\hline & hsa04350:TGF-beta signaling pathway & 4 & 0.056 & $\begin{array}{l}\text { ENST00000416274, ENST00000224764, ENST00000394092, } \\
\text { ENST00000401753 }\end{array}$ \\
\hline & hsa00510:N-Glycan biosynthesis & 3 & 0.077 & ENST00000393487, ENST00000413355, ENST00000250498 \\
\hline \multirow[t]{2}{*}{ Beta(1,1) } & hsa00830:Retinol metabolism & 6 & 0.002 & $\begin{array}{l}\text { ENST00000335125, ENST00000394887, ENST00000001146, } \\
\text { ENST00000432060, ENST00000412271, ENST00000336356 }\end{array}$ \\
\hline & hsa04350:TGF-beta signaling pathway & 4 & 0.056 & $\begin{array}{l}\text { ENST00000416274, ENST00000224764, ENST00000394092, } \\
\text { ENST00000401753 }\end{array}$ \\
\hline
\end{tabular}

A increased the ENST00000428597 expression in our study. In addition to SNP rs7865618, we also examined five other CAD-associated SNPs and found that two of them were associated with ANRIL expression at a nominal level ( $\mathrm{p}$ value $<0.05$ ) and the other three also showed a nonsignificant trend for association. The directions of the effects were the same for all of the CAD risk variants we examined in our study. This replicated the previous report that the same transcript was increased in the carriers of the risk haplotype in peripheral blood mononuclear cells and whole blood cells [25]. However, Jarinova et al. also specifically investigated the relationship between CAD risk variants and different splicing variants of ANRIL and reported that the CAD risk variant was associated with a decreased amount of the same long variant in whole blood cells [21]. Congrains et al. did not observe a strong association between the risk variants and the ANRIL long transcript in vascular smooth muscle cells [26].

One possible reason that the directions of effects are inconsistent is that these studies were performed in different tissues and have used different assays to target different exons. The transcript ENST00000428597 was the longest transcript of the gene ANRIL, which includes all 19 exons. Our study used the Affymetrix Human Exon 1.0 ST Array, which was designed to measure every exon in the gene and then summarize them into transcript level measurements based on known transcript composition. The other three studies used quantitative reverse transcription polymerase chain reaction assays with different probes in their studies (Holdt et al. targeted exon 18-19, Jarinova et al. targeted exon 15-16, Congrains targeted exon 17-18) [21,25,26]. As shown by Folkersen et al., ANRIL has many novel transcript variants depending by the tissue type [23]. Thus it is possible that different transcripts were measured in different studies or the same transcript behaved differently in different tissues and environments. A recent functional study suggests that the CAD risk variants promote ANRIL expression by disrupting STAT1 binding [39]. Nonetheless, taken together these results along with the fact that ANRIL is expressed in cells and tissues that are affected by atherosclerosis $[27,40]$ and are correlated with atherosclerosis severity $[25,26]$, they suggest that $A N R I L$ plays an important role in mediating the genetic effect of the Chr9p21 region on the susceptibility of atherosclerosis.

There are several ways that the noncoding RNA ANRIL can alter expression of associated protein coding genes, which include RNA interference, gene silencing, chromatin remodeling, and DNA methylation [41]. Recent studies on animals found that ANRIL knockdowns showed a substantial reduction of cellular proliferation, which is known to play an important role in atherosclerosis [42,43]. The important role of ANRIL on cell growth has also been observed in human vascular smooth muscle cells [26,27]. Other evidence suggests that ANRIL may also affect various genes by trans effect [44-46]. Further studies are needed to clarify the mechanism involved.

The present study is the first one that has focused on the trans effect of the variants in the Chr9p21 region across the whole genome. SKAT is a powerful SNP set association test, which requires very few assumptions [31]. We used this test to screen the whole genome and found that five transcripts from five genes were at least nominally associated with the Chr9p21 region. We also examined the association between the transcripts identified via trans analysis and single SNPs within the Chr9p21 region, and we found several trans-eQTL SNPs, including both rare and common variants. The common trans-eQTL SNP rs4977756 was in the same linkage disequilibrium block with known CAD risk variants, and its association with DHRS9 was supported by the same signal observed from the same tissue in the HapMap CEU sample. Although the other rare trans-eQTL SNPs were not available in the public database, we did examine the relationship between gene expression in our identified trans-effected genes and common CAD risk SNPs in the Chr9p21 region, and we found quite a few trans-eQTL SNPs. This provides evidence that the Chr9p21 region is indeed associated with the top trans-effected genes identified by 
SKAT. Nonetheless, studies with rare variants and larger sample size are needed to validate our findings.

Among the trans-effected genes identified by SKAT, the most well-known gene is $A B C A 1$, which is also located on chromosome 9. It encodes a membrane-associated protein that is a member of the superfamily of ATP-binding cassette $(A B C)$ transporters. The $A B C$ protein transports various molecules across membranes and has been shown to be an important regulator for cholesterol efflux from peripheral cells, and mutations in $A B C A 1$ contribute to low HDL [47-49]. It is known that low HDL cholesterol is an independent risk factor for CAD [50]. Given its role in lowering HDL cholesterol, loss of function mutations in $A B C A 1$ would increase CAD risk. This supposition has been supported by the observation that the mutations in $A B C A 1$ are associated with coronary artery calcification, a subclinical trait of atherosclerosis [51] as well as CAD [52]. The function of $A B C A 1$ in the development of atherosclerosis has also been confirmed in animals. $A B C A 1$ knockout mice develop larger atherosclerotic lesions, and overexpression of $A B C A 1$ inhibits the progression of atherosclerotic lesions [53-55]. It was suggested that $A B C A 1$ protects against atherosclerosis by removing cholesterol from macrophages in the artery wall and therefore prevents the formation of foam cells, which are critical for the formation of atherosclerotic plaque [56,57]. Though the Chr9p21 region has been implicated in the etiology of atherosclerosis for a long time, this is the first time that we have observed an association between this chromosomal region and a functional gene for atherosclerosis. Understanding the cellular mechanism underlying this association is a very important next step to advancing our understanding of the relationship between Chr9p21 and atherosclerosis.

The four other genes identified as significantly associated with the Chr9p21 region are CASP14, EIF1AY, DUT and DHRS9. CASP14 plays a central role in the execution phase of cell apoptosis and is overexpressed in tumors [58]. EIF1AY is on the Y chromosome and is overexpressed in men who have experienced a stroke [59]. DUT encodes an essential enzyme of nucleotide metabolism, which is vital to DNA replication [60]. DHRS9 encodes an enzyme that is involved in the metabolism of retinol [61]. Very little is known about these genes in terms of their roles in CAD, so future studies will be necessary.

Since the transcripts across the genome are highly correlated, applying a stringent $\mathrm{p}$-value cutoff to trans-effect analyses may be too conservative. Gene enrichment testing is a powerful way to reveal potential influential transcripts and pathways. According to the gene set analysis, the trans-regulated genes predicted by $\operatorname{Beta}(1,1)$ and $\operatorname{Beta}(0.5,0.5)$ are enriched in several pathways. Retinol metabolism, the top pathway, has shown to have important effects on atherogenesis, endothelial function, vascular calcification and lipid metabolism [62]. It is also correlated with alcoholic liver disease in which alcoholic patients display hepatic total retinol levels that are progressively lower with increasing severity of liver disease [63]. Notably, DHRS9 has two transcripts within the nominally significant transcripts identified by the SKAT test, increasing the credibility that this gene shows a true trans-effect. $A D H 1$, one class of alcohol dehydrogenase, catalyzes the oxidation of alcohols to aldehydes and also metabolizes retinol, hydroxysteroids, and lipid peroxidation products. It affects cardiovascular function by regulating alcohol digestion as well as via a direct effect on the vasculature [64]. Increased expression of CYP26B1 may aggravate atherosclerosis [65].

$\mathrm{N}$-glycan biosynthesis is another pathway that was enriched in the gene set analysis. $\mathrm{N}$-glycans located at the surface of the endothelium are responsible for interactions with monocytes. A previous study has demonstrated that $\mathrm{N}$-glycans are increased on the endothelial cell surface at sites of early human lesion development and are effectors of monocyte adhesion during atherogenesis [66]. The MAN1A2 gene, which was in this gene set, was reported to be down-regulated by TNF $\alpha$ and is part of an inflammatory mechanism in the pathological process of atherosclerosis.

Activation of the TGF- $\beta$ pathway is well recognized as the most important feature in the pathogenesis of cardiac remodeling and fibrosis. It also plays a crucial role in the regulation of vascular function. A series of papers reveals the effects of TGF- $\beta$ signals in angiogenesis and atherosclerosis [67]. BMPR1A and SMAD1 in our gene set are two core components in BMP pathway, which has shown to be related to pulmonary arterial hypertension and vascular calcification. These results all suggest that the risk variants of $\mathrm{CAD}$ in the Chr9p21 region may exert the trans effect by modulating the expression of genes responsible for critical functions involved in CAD.

There are a few limitations of the current study. First, as there is a considerable amount of gene-expression variation among populations [68], these results may not be applicable to other populations. For example, although the GENOA sample was a community-based sample of sibships selected on hypertension criteria, the frequency of effects could be different from other populations or a representative population-based sample of unrelated individuals. However, our previous contributions to studies in this region do not indicate significant allele frequency differences from other population-based epidemiological samples of unrelated non-Hispanic whites [69]. Second, transformed beta-lymphocytes were used in this study, and thus our results cannot be directly applied to other tissues in the in vivo environment. Third, our sample size is relatively small for a transcriptome-wide association analysis. 
Given the increased availability of both SNP data and gene expression profiles in epidemiologic samples, investigating both cis and trans effects to characterize potentially functional elements in genomic regions identified by GWAS is an important area of research. Considering the limitations of our particular study, future epidemiologic studies with larger sample sizes and more ethnicallydiverse populations are needed to replicate our findings. In addition, gene expression analyses with rare variants that are conducted in heart related tissue are another important next step for replication. However, whole genome scale transcriptome association analysis only serves as a screening procedure to provide potential targets for the research community. Thus, functional studies are also needed to examine how genetic variation in the Chr9p21 region plays a role in the etiology of CAD.

\section{Conclusions}

In conclusion, we used a novel SNP-set based method to specifically examine the genetic effect of the CAD risk variants of the Chr9p21 region on the transcript level gene expression across the whole genome. We replicated the previous finding that the ANRIL long variant is highly associated with the CAD risk variants. Moreover, we provide the first evidence that variation in the Chr9p21 region is associated with important functional genes and pathways, which play an important role in the progression of atherosclerosis. Taken together, this suggests that DNA sequence variation in the Chr9p21 region may affect CAD through both cis and trans mechanisms.

\section{Additional files}

Additional file 1: Table S1. Four SNPs in the Chr9p21 region associated with all four top transcripts identified by SKAT_Beta(1,25).

Additional file 2: Figure $\mathbf{S 1}$. The mRNA expression ( $\log _{2}$ scale) of the top 5 trans-effected transcripts (identified by SKAT) was plotted against each genotypes for the corresponding top SNPs (shown in red). Boxplot was added on top of each scatterplot to show the median and interquartile range of the expression level for each genotypes (shown in black). The five plots are for gene A: DUT (ENST00000331200), B: EIF1AY (ENST00000361365), C: CASP14 (ENST00000427043), D: ABCA1 (ENST00000374736) and E: DHRS9 (ENST00000412271).

\section{Abbreviations}

GWAS: Genome-wide association studies; GENOA: The Genetic Epidemiology Network of Arteriopathy; SNP: Single nucleotide polymorphism;

CAD: Coronary artery disease; eQTL: Expression quantitative trait loci.

\section{Competing interests}

The authors declare that they have no competing interests.

\section{Authors' contributions}

WZ participated in the design of the study, performed the statistical analysis, interpreted the results and drafted the manuscript. JAS participated in the design of the study, was involved in the interpretation of the results, and contributed critically to manuscript review and revision. GM performed the gene enrichment analysis and helped to draft the manuscript. WS helped with the look up work pertaining to publicly-available eQTL databases. MF, PAP, and
STT performed critical review of the manuscript. SLK conceived of the study, participated in its design, and was involved in the interpretation of the results. All authors read and approved the final manuscript.

\section{Acknowledgement}

We would like to thank all the GENOA participants. In addition, we are grateful for the generous support from the National Heart, Lung and Blood Institute (NHLBI) and the National Institute on Neurological Disorders and Stroke (NINDS) of the National Institutes of Health $(\mathrm{NIH})$ through grants HL100245, HL087660, HL119443 and NS041558.

\section{Author details}

'Department of Epidemiology, University of Michigan, Ann Arbor, MI, USA. ${ }^{2}$ Institute of Molecular Medicine and Human Genetics Center, University of Texas Health Science Center, Houston, TX, USA. ${ }^{3}$ Department of Epidemiology, Rollins School of Public Health, Emory University, Atlanta, GA, USA. ${ }^{4}$ Division of Nephrology and Hypertension, Department of Medicine, Mayo Clinic, Rochester, MN, USA.

Received: 18 December 2014 Accepted: 24 April 2015 Published online: 10 May 2015

\section{References}

1. Kochanek K, Xu J, Murphy SL, Miniño AM, Kung H. Deaths: Preliminary Data for. Natl Vital Stat Rep. 2009;2011:59(4).

2. Roger VL, Go AS, Lloyd-Jones DM, Adams RJ, Berry JD, Brown TM, et al. American heart association statistics committee and stroke statistics subcommittee: heart disease and stroke statistics-2011 update: a report from the american heart association. Circulation. 2011;123(4):e18-209.

3. Go AS, Mozaffarian D, Roger VL, Benjamin EJ, Berry JD, Blaha MJ, et al. Executive summary: heart disease and stroke statistics-2014 update: a report from the american heart association. Circulation. 2014;129(3):399-410.

4. Peyser PA. Genetic epidemiology of coronary artery disease. Epidemiol Rev. 1997;19(1):80-90.

5. Wellcome Trust Case Control Consortium. Genome-wide association study of 14,000 cases of seven common diseases and 3,000 shared controls. Nature. 2007:447(7145):661-78.

6. Samani NJ, Erdmann J, Hall AS, Hengstenberg C, Mangino M, Mayer B, et al. WTCCC and the cardiogenics consortium: genomewide association analysis of coronary artery disease. N Engl J Med. 2007;357(5):443-53.

7. Helgadottir A, Thorleifsson G, Manolescu A, Gretarsdottir S, Blondal T, Jonasdottir A, et al. A common variant on chromosome 9p21 affects the risk of myocardial infarction. Science. 2007;316(5830):1491-3.

8. Ye S, Willeit J, Kronenberg F, Xu Q, Kiechl S. Association of genetic variation on chromosome 9p21 with susceptibility and progression of atherosclerosis: a population-based, prospective study. J Am Coll Cardiol. 2008;52(5):378-84.

9. Schunkert H, Konig IR, Kathiresan S, Reilly MP, Assimes TL, Holm H, et al. Large-scale association analysis identifies 13 new susceptibility loci for coronary artery disease. Nat Genet. 2011;43(4):333-8.

10. Gori F, Specchia C, Pietri S, Crociati L, Barlera S, Franciosi M, et al. GISSI prevenzione investigators, SIBioC-GISSI prevenzione group: common genetic variants on chromosome 9p21 are associated with myocardial infarction and type 2 diabetes in an Italian population. BMC Med Genet. 2010;11:60.

11. Hiura Y, Fukushima Y, Yuno M, Sawamura H, Kokubo Y, Okamura T, et al. Validation of the association of genetic variants on chromosome 9p21 and $1 \mathrm{q} 41$ with myocardial infarction in a Japanese population. Circ J. 2008;72(8):1213-7.

12. Zhou L, Zhang X, He M, Cheng L, Chen Y, Hu FB, et al. Associations between single nucleotide polymorphisms on chromosome 9 p21 and risk of coronary heart disease in Chinese Han population. Arterioscler Thromb Vasc Biol. 2008;28(11):2085-9.

13. Assimes TL, Knowles JW, Basu A, lribarren C, Southwick A, Tang H, et al. Susceptibility locus for clinical and subclinical coronary artery disease at chromosome 9p21 in the multi-ethnic ADVANCE study. Hum Mol Genet. 2008;17(15):2320-8.

14. Smith JG, Melander O, Lovkvist $\mathrm{H}$, Hedblad B, Engstrom G, Nilsson P, et al. Common genetic variants on chromosome $9 p 21$ confers risk of ischemic stroke: a large-scale genetic association study. Circ Cardiovasc Genet. 2009;2(2):159-64. 
15. Bown MJ, Braund PS, Thompson J, London NJ, Samani NJ, Sayers RD. Association between the coronary artery disease risk locus on chromosome 9p21.3 and abdominal aortic aneurysm. Circ Cardiovasc Genet. 2008;1(1):39-42.

16. Thompson AR, Golledge J, Cooper JA, Hafez H, Norman PE, Humphries SE. Sequence variant on 9p21 is associated with the presence of abdominal aortic aneurysm disease but does not have an impact on aneurysmal expansion. Eur J Hum Genet. 2009;17(3):391-4.

17. Cluett C, McDermott MM, Guralnik J, Ferrucci L, Bandinelli S, Miljkovic I, et al. The 9p21 myocardial infarction risk allele increases risk of peripheral artery disease in older people. Circ Cardiovasc Genet. 2009;2(4):347-53.

18. Holdt LM, Teupser D. Recent studies of the human chromosome 9p21 locus, which is associated with atherosclerosis in human populations. Arterioscler Thromb Vasc Biol. 2012;32(2):196-206.

19. Gayther SA, Song H, Ramus SJ, Kjaer SK, Whittemore AS, Quaye L, et al. Ovarian cancer association consortium: tagging single nucleotide polymorphisms in cell cycle control genes and susceptibility to invasive epithelial ovarian cancer. Cancer Res. 2007;67(7):3027-35.

20. Pasmant E, Laurendeau I, Heron D, Vidaud M, Vidaud D, Bieche I. Characterization of a germ-line deletion, including the entire INK4/ARF locus, in a melanomaneural system tumor family: identification of ANRIL, an antisense noncoding RNA whose expression coclusters with ARF. Cancer Res. 2007;67(8):3963-9.

21. Jarinova O, Stewart AF, Roberts R, Wells G, Lau P, Naing T, et al. Functional analysis of the chromosome 9p21.3 coronary artery disease risk locus. Arterioscler Thromb Vasc Biol. 2009;29(10):1671-7.

22. Liu Y, Sanoff HK, Cho H, Burd CE, Torrice C, Mohlke KL, et al. INK4/ARF transcript expression is associated with chromosome 9p21 variants linked to atherosclerosis. PLoS One. 2009;4(4), e5027.

23. Folkersen L, Kyriakou T, Goel A, Peden J, Malarstig A, Paulsson-Berne G, et al. PROCARDIS consortia: Relationship between CAD risk genotype in the chromosome 9p21 locus and gene expression. Identification of eight new ANRIL splice variants. PLoS One. 2009;4(11), e7677.

24. Cunnington MS, Santibanez Koref M, Mayosi BM, Burn J, Keavney B. Chromosome 9p21 SNPs Associated with Multiple Disease Phenotypes Correlate with ANRIL Expression. PLoS Genet. 2010;6(4), e1000899.

25. Holdt LM, Beutner F, Scholz M, Gielen S, Gabel G, Bergert H, et al. ANRIL expression is associated with atherosclerosis risk at chromosome 9p21. Arterioscler Thromb Vasc Biol. 2010;30(3):620-7.

26. Congrains A, Kamide K, Oguro R, Yasuda O, Miyata K, Yamamoto E, et al. Genetic variants at the 9p21 locus contribute to atherosclerosis through modulation of ANRIL and CDKN2A/B. Atherosclerosis. 2012;220(2):449-55.

27. Motterle A, Pu X, Wood H, Xiao Q, Gor S, Ng FL, et al. Functional analyses of coronary artery disease associated variation on chromosome 9p21 in vascular smooth muscle cells. Hum Mol Genet. 2012;21(18):4021-9.

28. Price AL, Patterson N, Hancks DC, Myers S, Reich D, Cheung VG, et al. Effects of cis and trans genetic ancestry on gene expression in African Americans. PLoS Genet. 2008;4(12), e1000294.

29. Holdt LM, Teupser D. From genotype to phenotype in human atherosclerosisrecent findings. Curr Opin Lipidol. 2013;24(5):410-8.

30. Daniels PR, Kardia SL, Hanis CL, Brown CA, Hutchinson R, Boerwinkle E, et al. Genetic epidemiology network of arteriopathy study: familial aggregation of hypertension treatment and control in the genetic epidemiology network of arteriopathy (GENOA) study. Am J Med. 2004;116(10):676-81.

31. Wu MC, Lee S, Cai T, Li Y, Boehnke M, Lin X. Rare-variant association testing for sequencing data with the sequence kernel association test. Am J Hum Genet. 2011;89(1):82-93.

32. Champtiaux N, Kalivas PW, Bardo MT. Contribution of dihydro-beta-erythroidine sensitive nicotinic acetylcholine receptors in the ventral tegmental area to cocaine-induced behavioral sensitization in rats. Behav Brain Res. 2006;168(1):120-6.

33. Chen P, Lepikhova T, Hu Y, Monni O, Hautaniemi S. Comprehensive exon array data processing method for quantitative analysis of alternative spliced variants. Nucleic Acids Res. 2011;39(18), e123.

34. Koch W, Turk S, Erl A, Hoppmann P, Pfeufer A, King L, et al. The chromosome 9p21 region and myocardial infarction in a European population. Atherosclerosis. 2011;217(1):220-6.

35. Madsen BE, Browning SR. A groupwise association test for rare mutations using a weighted sum statistic. PLoS Genet. 2009;5(2), e1000384.

36. da Huang W, Sherman BT, Lempicki RA. Systematic and integrative analysis of large gene lists using DAVID bioinformatics resources. Nat Protoc. 2009;4(1):44-57.
37. Yang TP, Beazley C, Montgomery SB, Dimas AS, Gutierrez-Arcelus M, Stranger BE, et al. Genevar: a database and Java application for the analysis and visualization of SNP-gene associations in eQTL studies. Bioinformatics. 2010;26(19):2474-6.

38. GTEx Consortium. The genotype-tissue expression (GTEx) project. Nat Genet. 2013;45(6):580-5.

39. Harismendy O, Notani D, Song X, Rahim NG, Tanasa B, Heintzman N, et al. 9p21 DNA variants associated with coronary artery disease impair interferon-gamma signalling response. Nature. 2011;470(7333):264-8.

40. Broadbent HM, Peden JF, Lorkowski S, Goel A, Ongen H, Green F, et al. PROCARDIS consortium: Susceptibility to coronary artery disease and diabetes is encoded by distinct, tightly linked SNPs in the ANRIL locus on chromosome 9p. Hum Mol Genet. 2008;17(6):806-14.

41. Amaral PP, Dinger ME, Mercer TR, Mattick JS. The eukaryotic genome as an RNA machine. Science. 2008;319(5871):1787-9.

42. Kotake Y, Nakagawa T, Kitagawa K, Suzuki S, Liu N, Kitagawa M, et al. Long non-coding RNA ANRIL is required for the PRC2 recruitment to and silencing of p15(INK4B) tumor suppressor gene. Oncogene. 2011;30(16):1956-62.

43. Yap KL, Li S, Munoz-Cabello AM, Raguz S, Zeng L, Mujtaba S, et al. Molecular interplay of the noncoding RNA ANRIL and methylated histone $\mathrm{H} 3$ lysine 27 by polycomb CBX7 in transcriptional silencing of INK4a. Mol Cell. 2010;38(5):662-74.

44. Sato K, Nakagawa H, Tajima A, Yoshida K, Inoue I. ANRIL is implicated in the regulation of nucleus and potential transcriptional target of E2F1. Oncol Rep. 2010;24(3):701-7.

45. Congrains A, Kamide K, Katsuya T, Yasuda O, Oguro R, Yamamoto K, et al. CVDassociated non-coding RNA, ANRIL, modulates expression of atherogenic pathways in VSMC. Biochem Biophys Res Commun. 2012;419(4):612-6.

46. Holdt LM, Hoffmann S, Sass K, Langenberger D, Scholz M, Krohn K, et al. Alu elements in ANRIL non-coding RNA at chromosome 9p21 modulate atherogenic cell functions through trans-regulation of gene networks. PLoS Genet. 2013;9(7), e1003588.

47. Frikke-Schmidt R, Nordestgaard BG, Jensen GB, Tybjaerg-Hansen A. Genetic variation in $A B C$ transporter $A 1$ contributes to $H D L$ cholesterol in the general population. J Clin Invest. 2004;114(9):1343-53.

48. Cohen JC, Kiss RS, Pertsemlidis A, Marcel YL, McPherson R, Hobbs HH. Multiple rare alleles contribute to low plasma levels of $\mathrm{HDL}$ cholesterol. Science. 2004;305(5685):869-72.

49. Soufi M, Ruppert $V$, Kurt B, Schaefer JR. The impact of severe LDL receptor mutations on SREBP-pathway regulation in homozygous familial hypercholesterolemia (FH). Gene. 2012;499(1):218-22.

50. Gordon T, Castelli WP, Hjortland MC, Kannel WB, Dawber TR. High density lipoprotein as a protective factor against coronary heart disease. The Framingham Study. Am J Med. 1977;62(5):707-14.

51. Benton JL, Ding J, Tsai MY, Shea S, Rotter Jl, Burke GL, et al. Associations between two common polymorphisms in the ABCA1 gene and subclinical atherosclerosis: Multi-Ethnic Study of Atherosclerosis (MESA). Atherosclerosis. 2007;193(2):352-60.

52. Tietjen I, Hovingh GK, Singaraja R, Radomski C, McEwen J, Chan E, et al. Increased risk of coronary artery disease in Caucasians with extremely low HDL cholesterol due to mutations in ABCA1, APOA1, and LCAT. Biochim Biophys Acta. 2012;1821(3):416-24.

53. van Eck M, Bos IS, Kaminski WE, Orso E, Rothe G, Twisk J, et al. Leukocyte ABCA1 controls susceptibility to atherosclerosis and macrophage recruitment into tissues. Proc Natl Acad Sci U S A. 2002;99(9):6298-303.

54. Van Eck M, Singaraja RR, Ye D, Hildebrand RB, James ER, Hayden MR, et al. Macrophage ATP-binding cassette transporter A1 overexpression inhibits atherosclerotic lesion progression in low-density lipoprotein receptor knockout mice. Arterioscler Thromb Vasc Biol. 2006;26(4):929-34.

55. Lammers B, Zhao Y, Hoekstra M, Hildebrand RB, Ye D, Meurs I, et al. Augmented atherogenesis in LDL receptor deficient mice lacking both macrophage ABCA1 and ApoE. PLoS One. 2011;6(10), e26095.

56. Jessup W, Gelissen IC, Gaus K, Kritharides L. Roles of ATP binding cassette transporters $A 1$ and $\mathrm{G} 1$, scavenger receptor $\mathrm{B}$ and membrane lipid domains in cholesterol export from macrophages. Curr Opin Lipidol. 2006:17(3):247-57.

57. Weissglas-Volkov D, Pajukanta P. Genetic causes of high and low serum HDL-cholesterol. J Lipid Res. 2010;51(8):2032-57.

58. Krajewska M, Kim H, Shin E, Kennedy S, Duffy MJ, Wong YF, et al. Tumorassociated alterations in caspase-14 expression in epithelial malignancies. Clin Cancer Res. 2005;11(15):5462-71. 
59. Tian Y, Stamova B, Jickling GC, Xu H, Liu D, Ander BP, et al. Y chromosome gene expression in the blood of male patients with ischemic stroke compared with male controls. Gend Med. 2012;9(2):68-75. e3.

60. McIntosh EM, Ager DD, Gadsden MH, Haynes RH. Human dUTP pyrophosphatase: CDNA sequence and potential biological importance of the enzyme. Proc Natl Acad Sci U S A. 1992;89(17):8020-4.

61. Soref CM, Di YP, Hayden L, Zhao YH, Satre MA, Wu R. Characterization of a novel airway epithelial cell-specific short chain alcohol dehydrogenase/ reductase gene whose expression is up-regulated by retinoids and is involved in the metabolism of retinol. J Biol Chem. 2001;276(26):24194-202.

62. Rhee EJ, Nallamshetty S, Plutzky J. Retinoid metabolism and its effects on the vasculature. Biochim Biophys Acta. 2012;1821(1):230-40.

63. Mansouri A, Demeilliers C, Amsellem S, Pessayre D, Fromenty B. Acute ethanol administration oxidatively damages and depletes mitochondrial dna in mouse liver, brain, heart, and skeletal muscles: protective effects of antioxidants. J Pharmacol Exp Ther. 2001;298(2):737-43.

64. Jelski W, Orywal K, Panek B, Gacko M, Mroczko B, Szmitkowski M. The activity of class I, II, III and IV of alcohol dehydrogenase (ADH) isoenzymes and aldehyde dehydrogenase (ALDH) in the wall of abdominal aortic aneurysms. Exp Mol Pathol. 2009;87(1):59-62.

65. Krivospitskaya O, Elmabsout AA, Sundman E, Soderstrom LA, Ovchinnikova O, Gidlof AC, et al. A CYP26B1 polymorphism enhances retinoic acid catabolism and may aggravate atherosclerosis. Mol Med. 2012;18:712-8.

66. Scott DW, Chen J, Chacko BK, Traylor Jr JG, Orr AW, Patel RP. Role of endothelial $\mathrm{N}$-glycan mannose residues in monocyte recruitment during atherogenesis. Arterioscler Thromb Vasc Biol. 2012:32(8): 5 51-9.

67. Pardali E, Ten Dijke P. TGFbeta signaling and cardiovascular diseases. Int J Biol Sci. 2012;8(2):195-213.

68. Storey JD, Madeoy J, Strout JL, Wurfel M, Ronald J, Akey JM. Gene-expression variation within and among human populations. Am J Hum Genet. 2007:80(3):502-9

69. O'Donnell CJ, Kavousi M, Smith AV, Kardia SL, Feitosa MF, Hwang SJ, et al. Genome-wide association study for coronary artery calcification with follow-up in myocardial infarction. Circulation. 2011;124(25):2855-64.

\section{Submit your next manuscript to BioMed Central and take full advantage of:}

- Convenient online submission

- Thorough peer review

- No space constraints or color figure charges

- Immediate publication on acceptance

- Inclusion in PubMed, CAS, Scopus and Google Scholar

- Research which is freely available for redistribution 